\title{
A General Approach to 3-n-Butyl-5-alkyl-indolizidines: Total Synthesis of (-)-Indolizidine 195B
}

\author{
Steven R. Angle* and Musong Kim \\ Department of Chemistry, University of California-Riverside, Riverside, CA 92521-0403 \\ steven.angle@wright.edu
}

Table of Contents:

Pages

General Information

$\mathrm{S} 2$

NMR and Specific Rotation Comparison of $\mathbf{1}$ to the literature precedent

S3

${ }^{1} \mathrm{H}$ and ${ }^{13} \mathrm{C}$ NMR Spectra for 1, 9, 10, 11, 12, 15, 16, 17, 18, 20, 21, 22, 23, 24, 25, and 26

S4-S42

Determination of diastereomer ratio of $\mathbf{1 5}$

S13

Determination of diastereomer ratio of $\mathbf{1 6}$

S18

Decoupling study of $\mathbf{1 8}$

S24

Determination of diastereomer ratio of 1 [(-)-Indolizidine 195B]

S41

i 
General Information: All reactions in non-aqueous solvents were carried out in flame dried or oven dried glassware under an atmosphere of nitrogen or argon. Ether and THF were distilled from sodium/benzophenone. Methylene chloride, methanol, toluene were distilled from $\mathrm{CaH}_{2}$. Acetic anhydride was distilled from $\mathrm{P}_{2} \mathrm{O}_{5}$.

Melting Points were determined on a capillary melting point apparatus. ${ }^{1} \mathrm{H}$ NMR and ${ }^{13} \mathrm{C}$ NMR spectra were recorded in $\mathrm{CDCl}_{3}, \mathrm{D}_{2} \mathrm{O}$ and $\mathrm{CD}_{3} \mathrm{OD}$ on a 300 or $400 \mathrm{MHz}$ NMR. Optical rotations were measured using the sodium D Line.

Thin layer chromatographies were performed on glass backed silica gel plates ( $250 \mathrm{~mm}$ thickness, with a $254 \mathrm{~nm}$ fluorescent indicator). Prep TLC was done on Aluminiumoxide $60 \mathrm{~F}_{254}$ neutral (Type E) plate with $0.2 \mathrm{~mm}$ thickness, $20 \mathrm{~cm}$ X $20 \mathrm{~cm}$. Flash chromatography was carried out using silica gel (230-400 mesh) with distilled solvents. HPLC was performed using a 60A Si 83-101-c column (4.6 mm ID). The following parameters were used for HPLC separation unless stated otherwise: flow rate $=0.5 \mathrm{~mL} / \mathrm{min}$; the appropriate mixture of hexanes/ethyl acetate was used. In all cases solvents were removed in vaccuo under reduced pressure with a rotary evaporator.

Capillary GC was carried out using an FID detector on a 25 m methyl silicone column. The following standard GC parameters were used unless indicated otherwise: flow rate $=60 \mathrm{~mL} / \mathrm{min}$; injector temp $=200{ }^{\circ} \mathrm{C}$; detector temperature $=280{ }^{\circ} \mathrm{C}$; temperature program $=40{ }^{\circ} \mathrm{C}$ to $280{ }^{\circ} \mathrm{C}$ at $15{ }^{\circ} \mathrm{C} / \mathrm{min}$, initial time $=2 \min$.

The molarity indicated for vinylmagnesium chloride was established by titration with $2,2^{\prime}$ bipyridyl/s-butyl alcohol in methylene chloride. ${ }^{35}$ Buffer solutions were prepared with $\mathrm{Na}_{2} \mathrm{HPO}_{4}$ and citric acid. ${ }^{36}$ Organic solvents were removed under reduced pressure (water aspirator) with a rotary evaporator. All reactions were run under an atmosphere of nitrogen in flame-dried glassware.

Mass spectra are reported as percent relative intensity to the base peak. 
NMR and Specific Rotation Comparison of 1 to literature precedent



${ }^{1}$ Lhommet, G.; Célimène, C.; Dhimane, H. Tetrahedron 1998, 54, 10457-10468.

${ }^{2}$ Literature ${ }^{1} \mathrm{H}$ NMR was performed using a $200 \mathrm{MHz}$ instrument; Observed was performed using a $300 \mathrm{MHz}$ instrument;

${ }^{3}$ Literature ${ }^{13} \mathrm{C}$ NMR was performed using a $50 \mathrm{MHz}$ instrument; Observed was performed using a $70 \mathrm{MHz}$ instrument. 
<smiles>OC[C@H]1CC=C[C@@H](CCCOC(F)(F)F)N1Cc1ccccc1</smiles>

${ }^{1} \mathrm{H} \mathrm{NMR}\left(300 \mathrm{MHz}, \mathrm{CDCl}_{3}\right)$

$\stackrel{\infty}{+}$






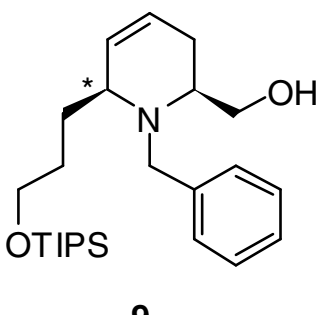

${ }^{13} \mathrm{C} \mathrm{NMR}\left(75 \mathrm{MHz}, \mathrm{CDCl}_{3}\right)$ 


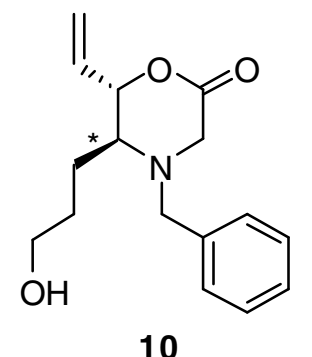

${ }^{1} \mathrm{H}$ NMR $\left(300 \mathrm{MHz}, \mathrm{CDCl}_{3}\right)$

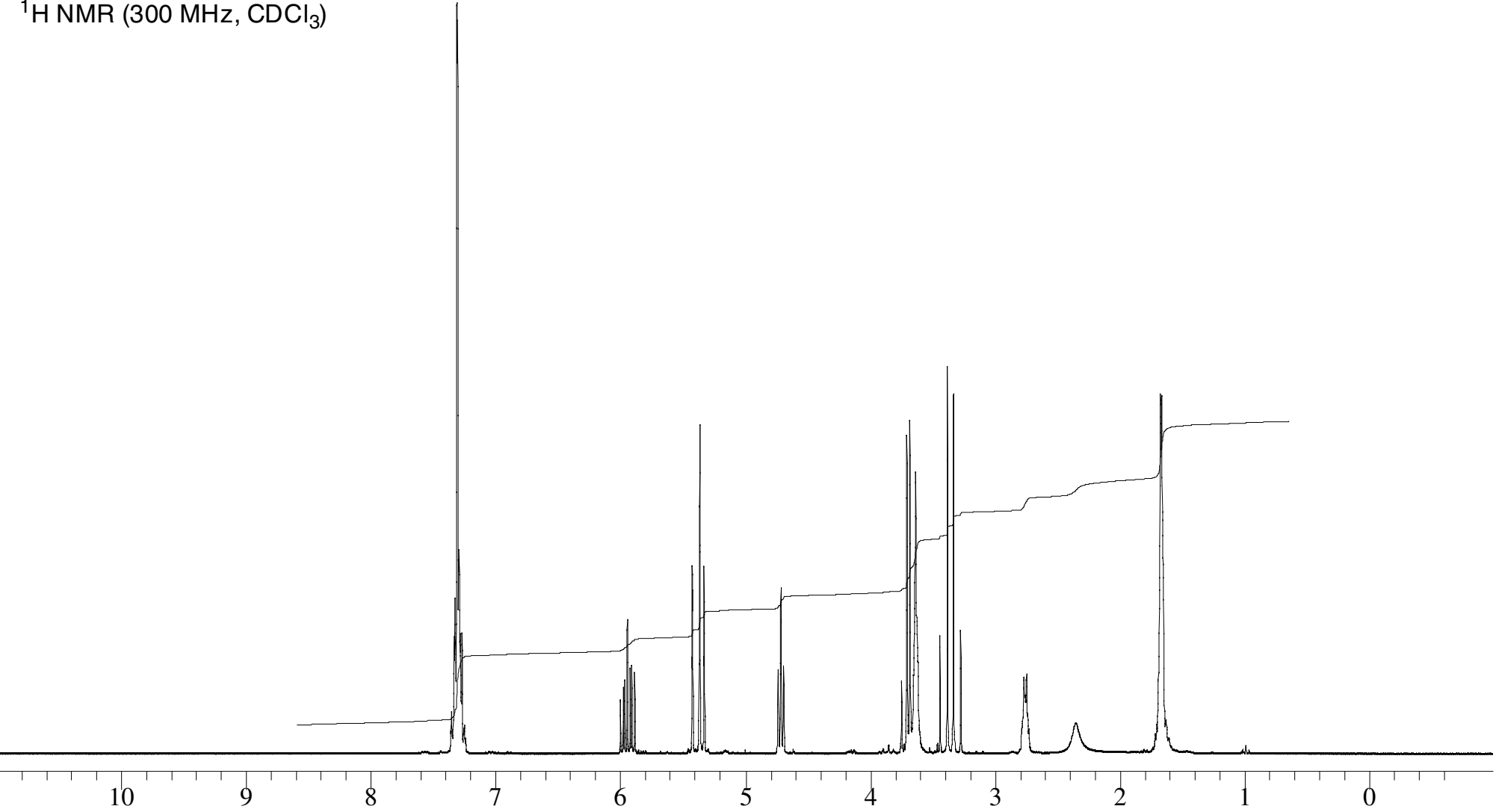




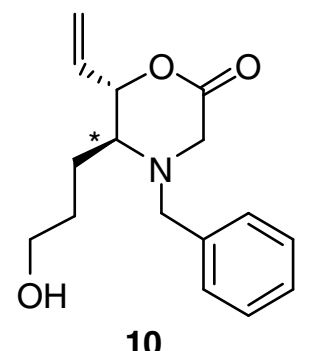

${ }^{13} \mathrm{C}$ NMR $\left(75 \mathrm{MHz}, \mathrm{CDCl}_{3}\right)$

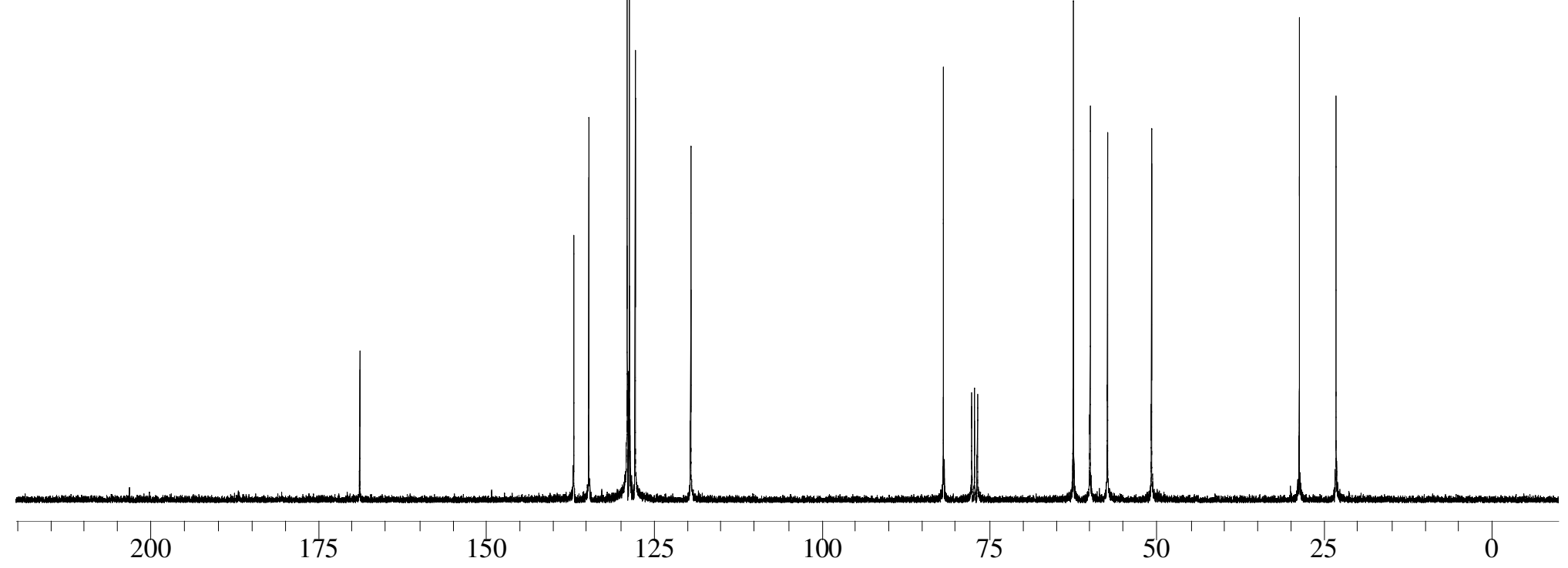






${ }^{1} \mathrm{H}$ NMR $\left(300 \mathrm{MHz}, \mathrm{CDCl}_{3}\right)$

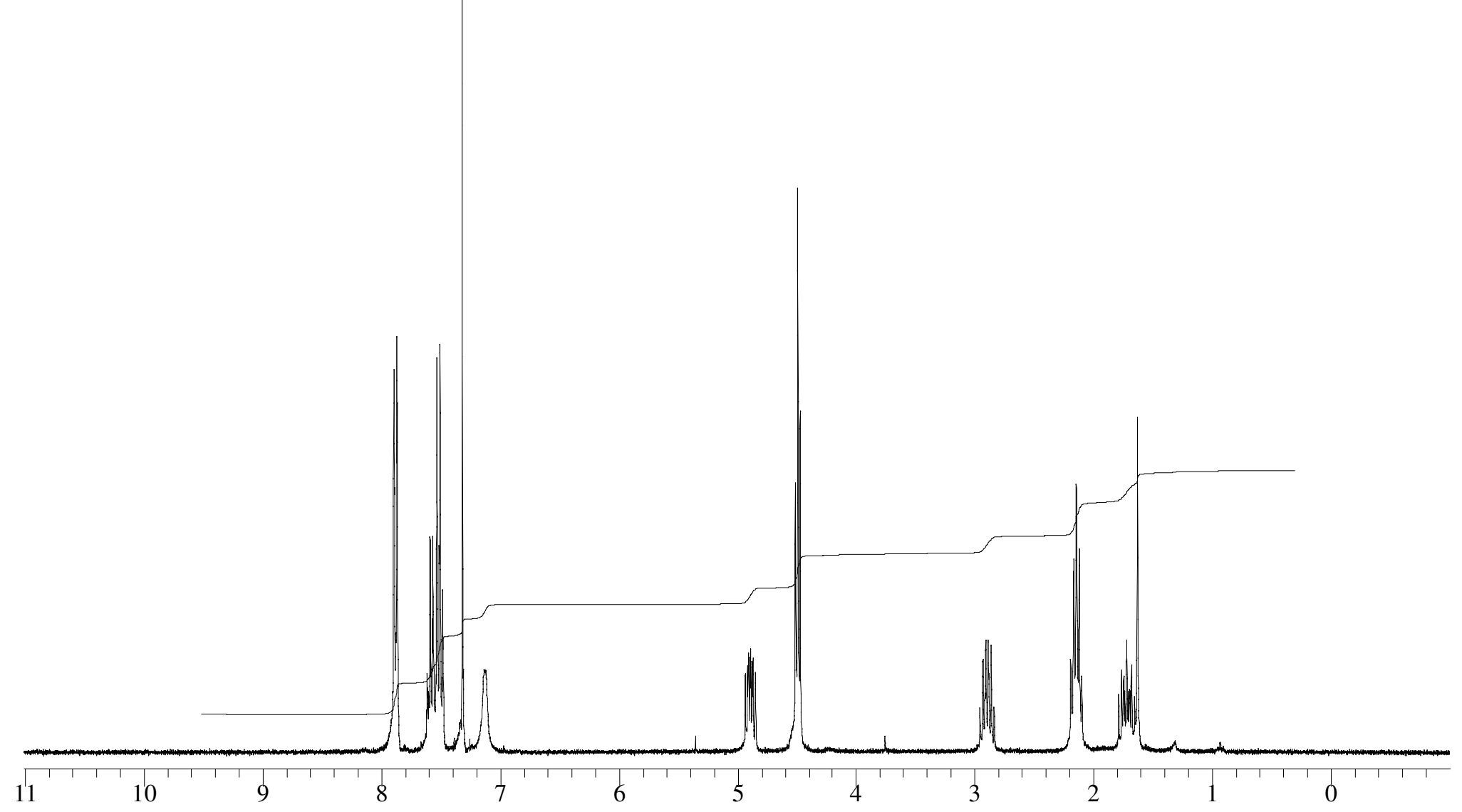




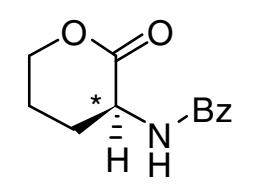

11

${ }^{13} \mathrm{C}$ NMR (75 MHz, $\left.\mathrm{CDCl}_{3}\right)$

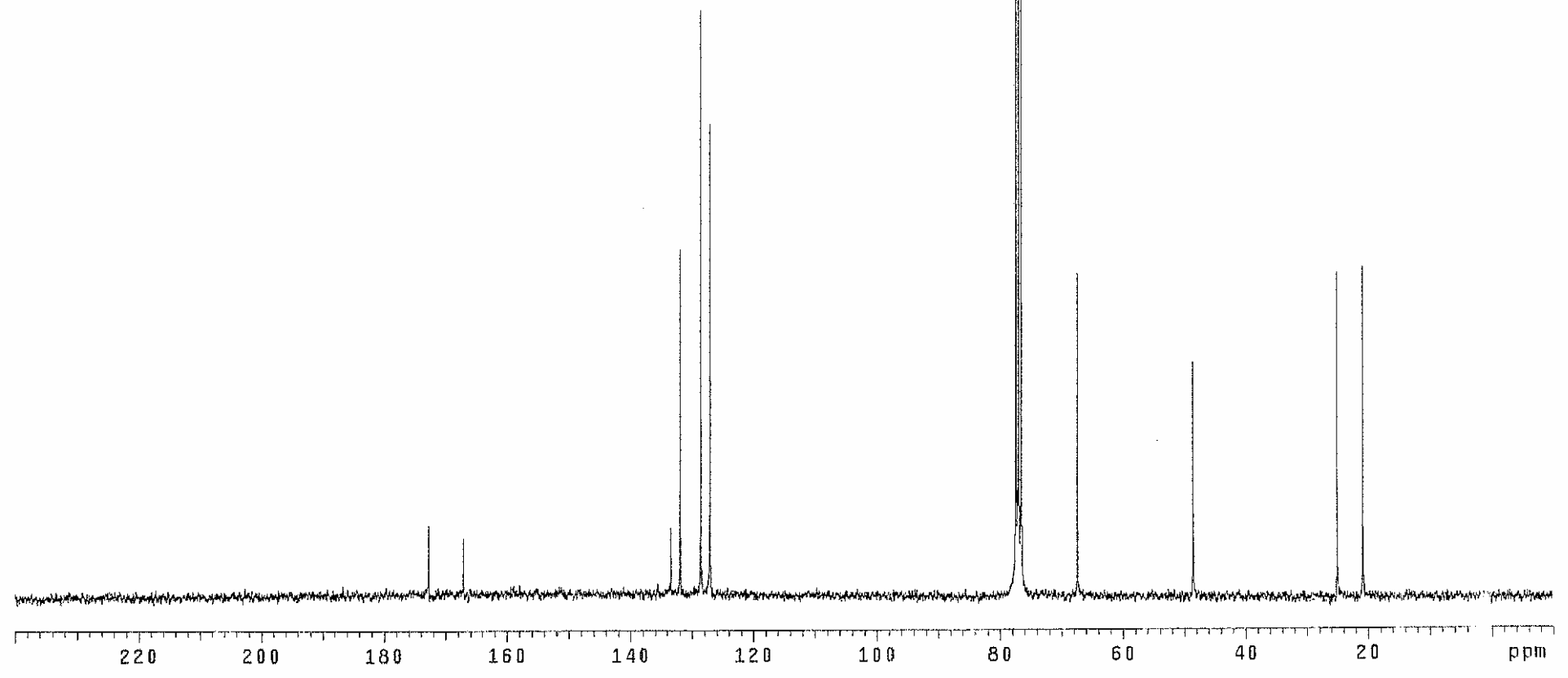




$$
\mathrm{CO}_{2} \mathrm{H}^{\mathrm{N}^{-} \mathrm{Bz}}
$$

12

${ }^{1} \mathrm{H}$ NMR (300 MHz, D 2 ) 


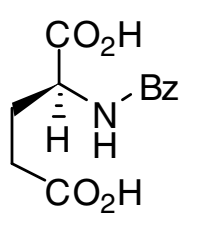

12

${ }^{13} \mathrm{C}$ NMR $\left(75 \mathrm{MHz}, \mathrm{D}_{2} \mathrm{O}\right)$

$\stackrel{\infty}{=}$






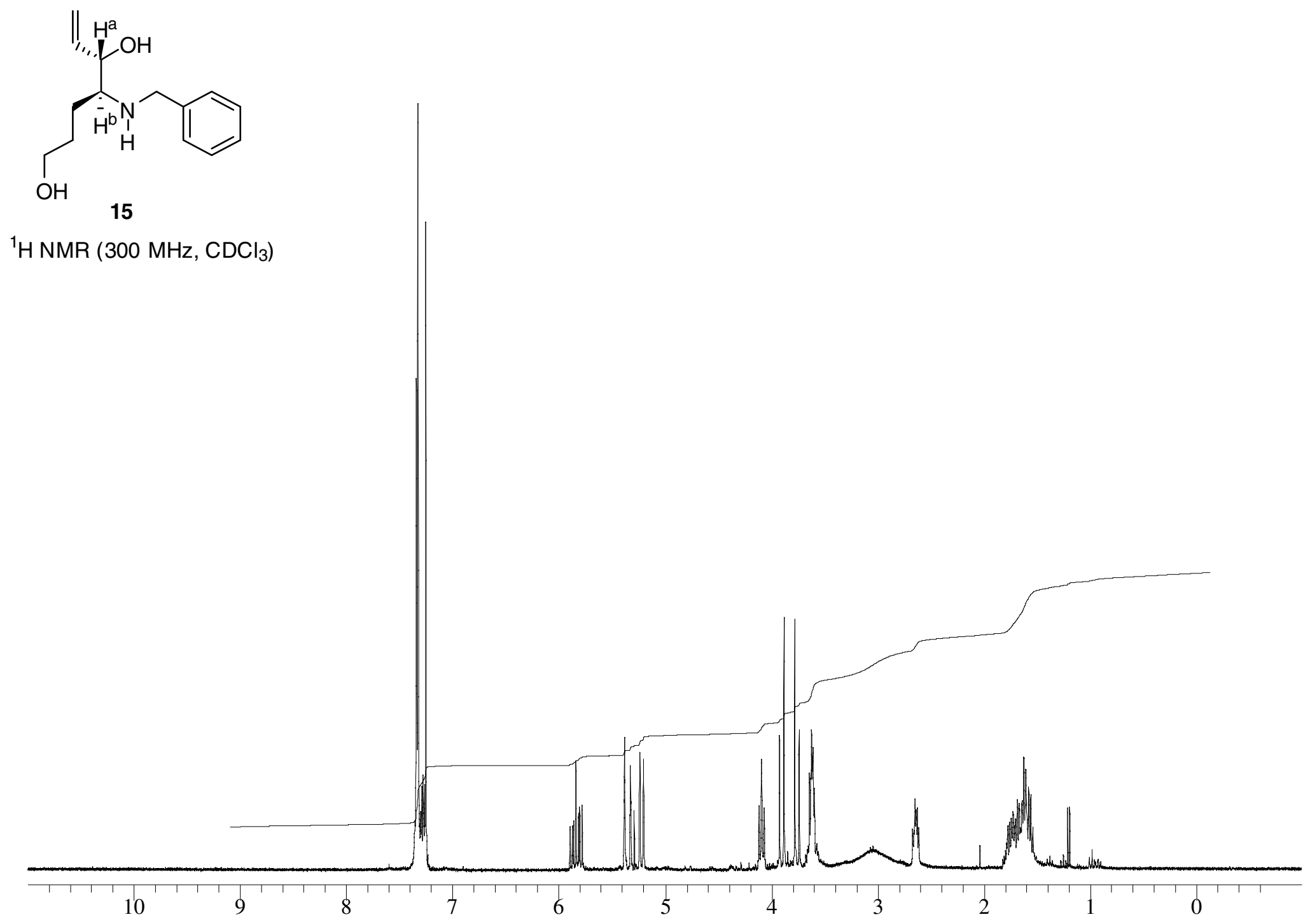




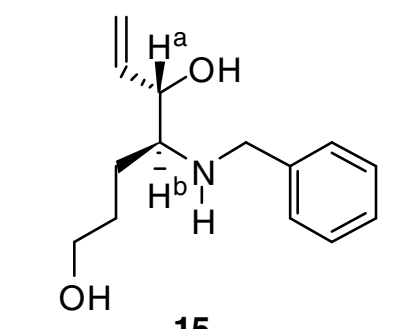

${ }^{1} \mathrm{H}$ NMR $\left(500 \mathrm{MHz}, \mathrm{CDCl}_{3}\right)$

$\frac{\omega}{\omega}$

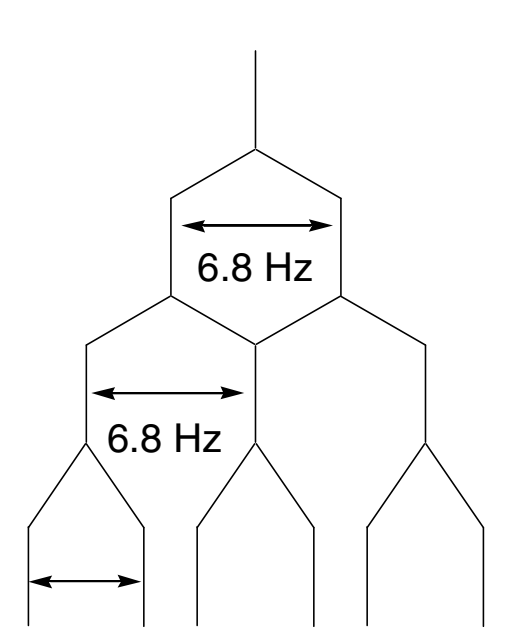

$3.9 \mathrm{~Hz}$
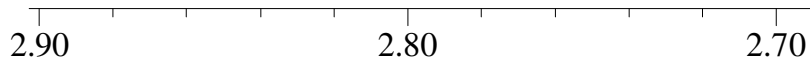

$\mathrm{H}^{\mathrm{b}}$

$\downarrow$

$\mathrm{J}=6.838$

2.90

2.80

2.70

2.60

2.50 


\section{Diastereomer ratio determination of the}

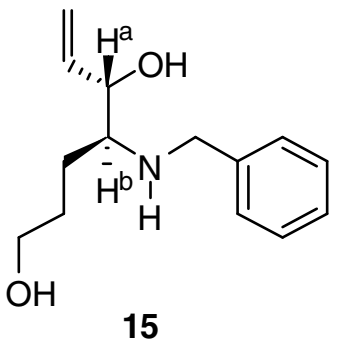

crude mixture of 15

$(\sim 8: 1)$

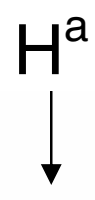

${ }^{1} \mathrm{H}$ NMR (300 MHz, CDCl ${ }_{3}$ )

$\stackrel{\infty}{\perp}$

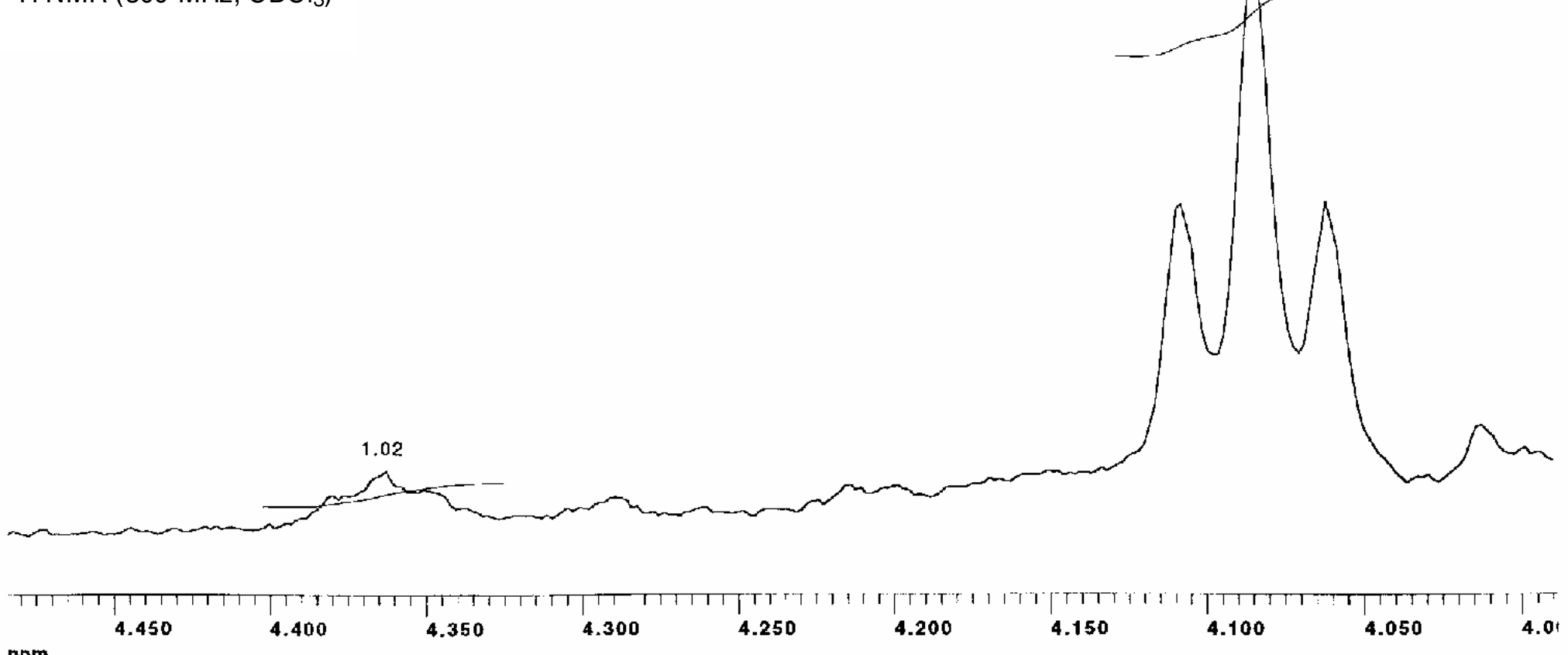




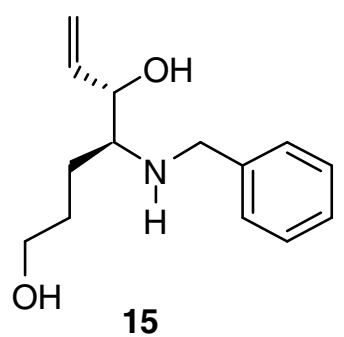

${ }^{13} \mathrm{C}$ NMR (75 MHz, $\mathrm{CDCl}_{3}$ )

$\stackrel{\infty}{\sim}$

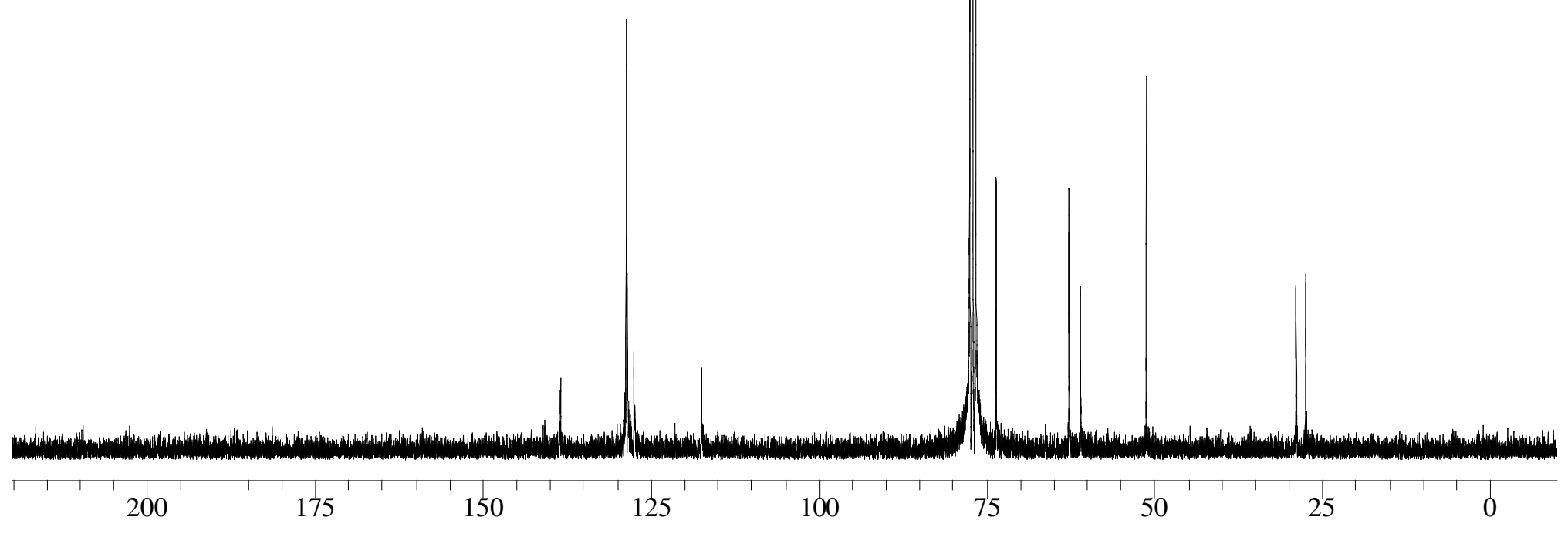




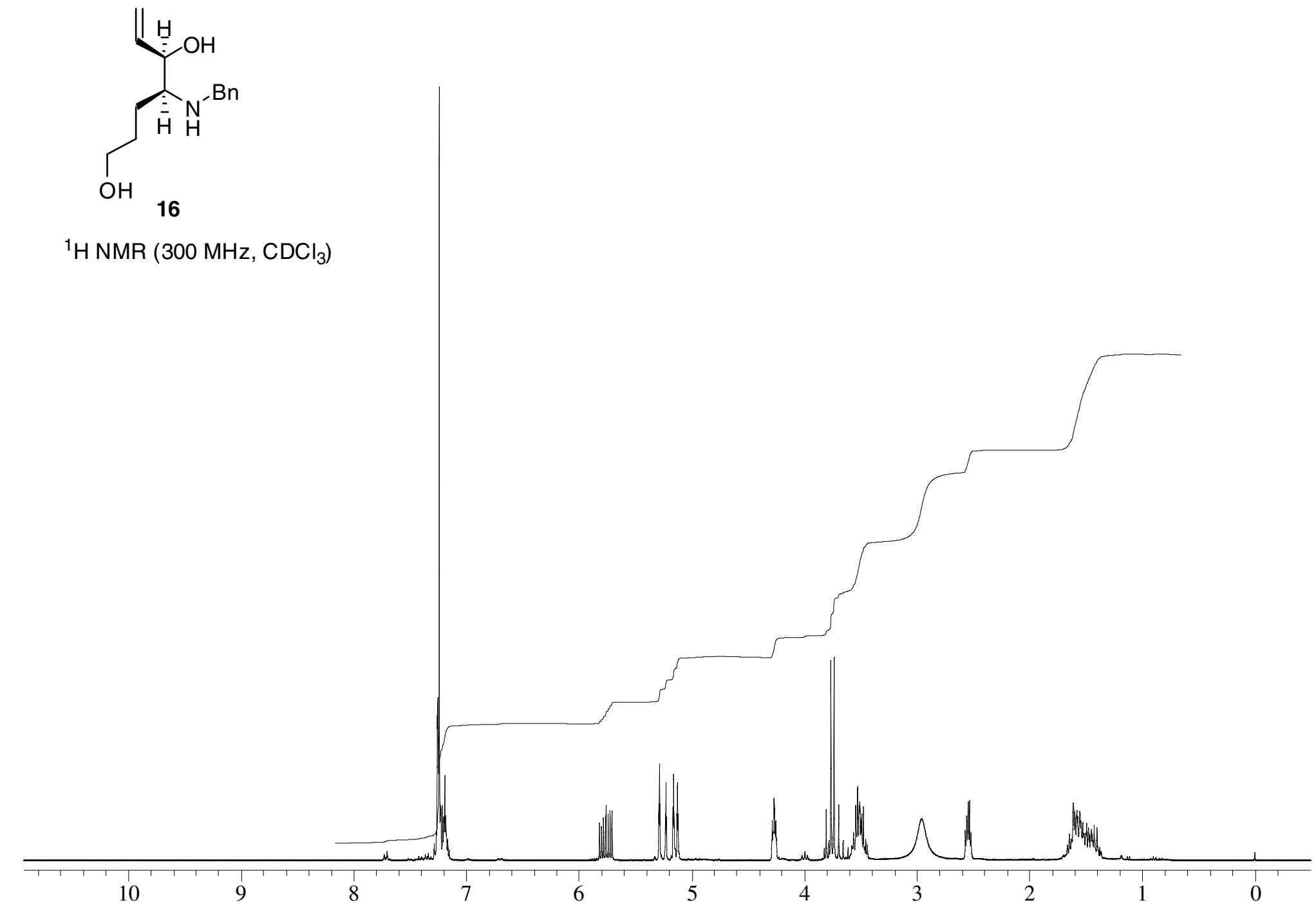

${ }^{1} \mathrm{H}$ NMR (300 MHz, $\mathrm{CDCl}_{3}$ )

$\frac{c}{a}$ 


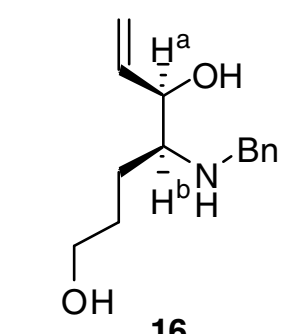

${ }^{1} \mathrm{H}$ NMR $\left(300 \mathrm{MHz}, \mathrm{CDCl}_{3}\right)$

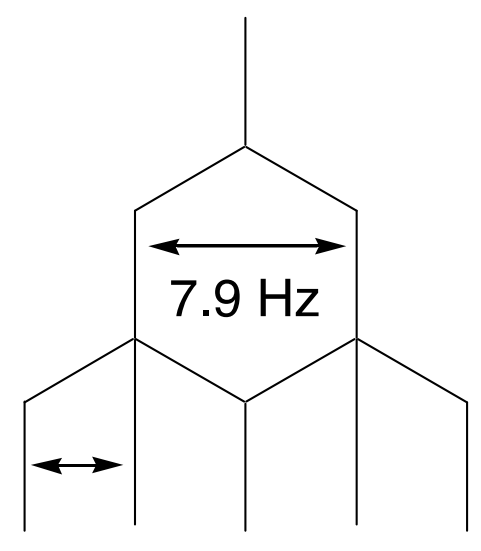

$3.9 \mathrm{~Hz}$

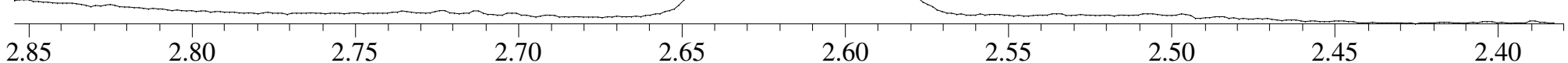




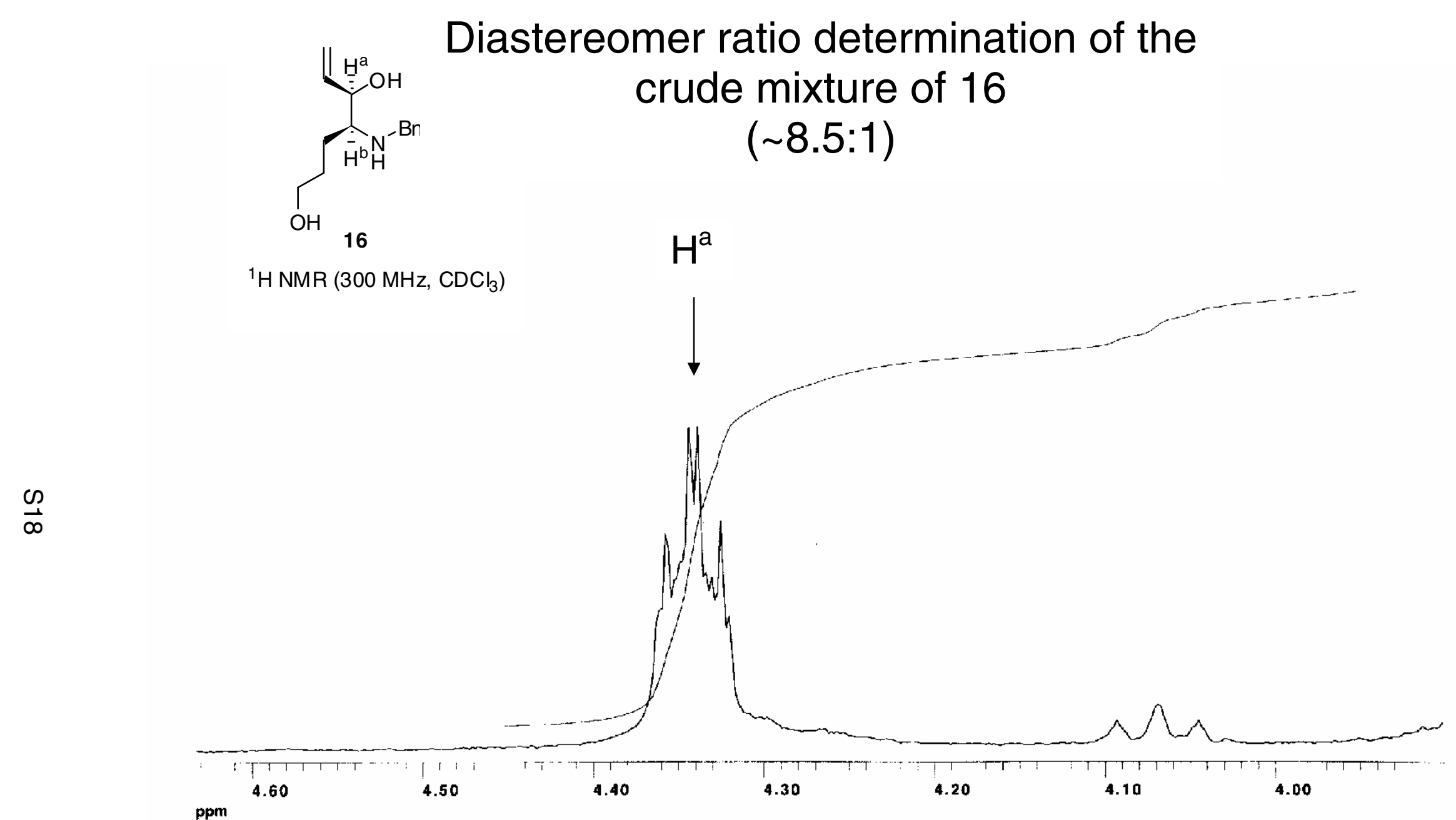




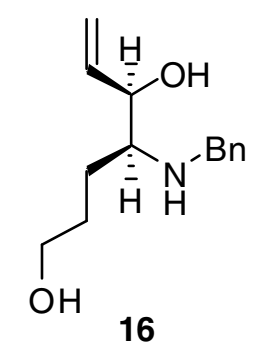

${ }^{13} \mathrm{C}$ NMR $\left(75 \mathrm{MHz}, \mathrm{CDCl}_{3}\right)$

$\stackrel{0}{0}$

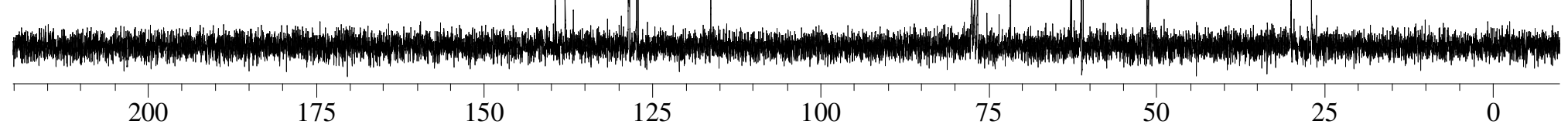




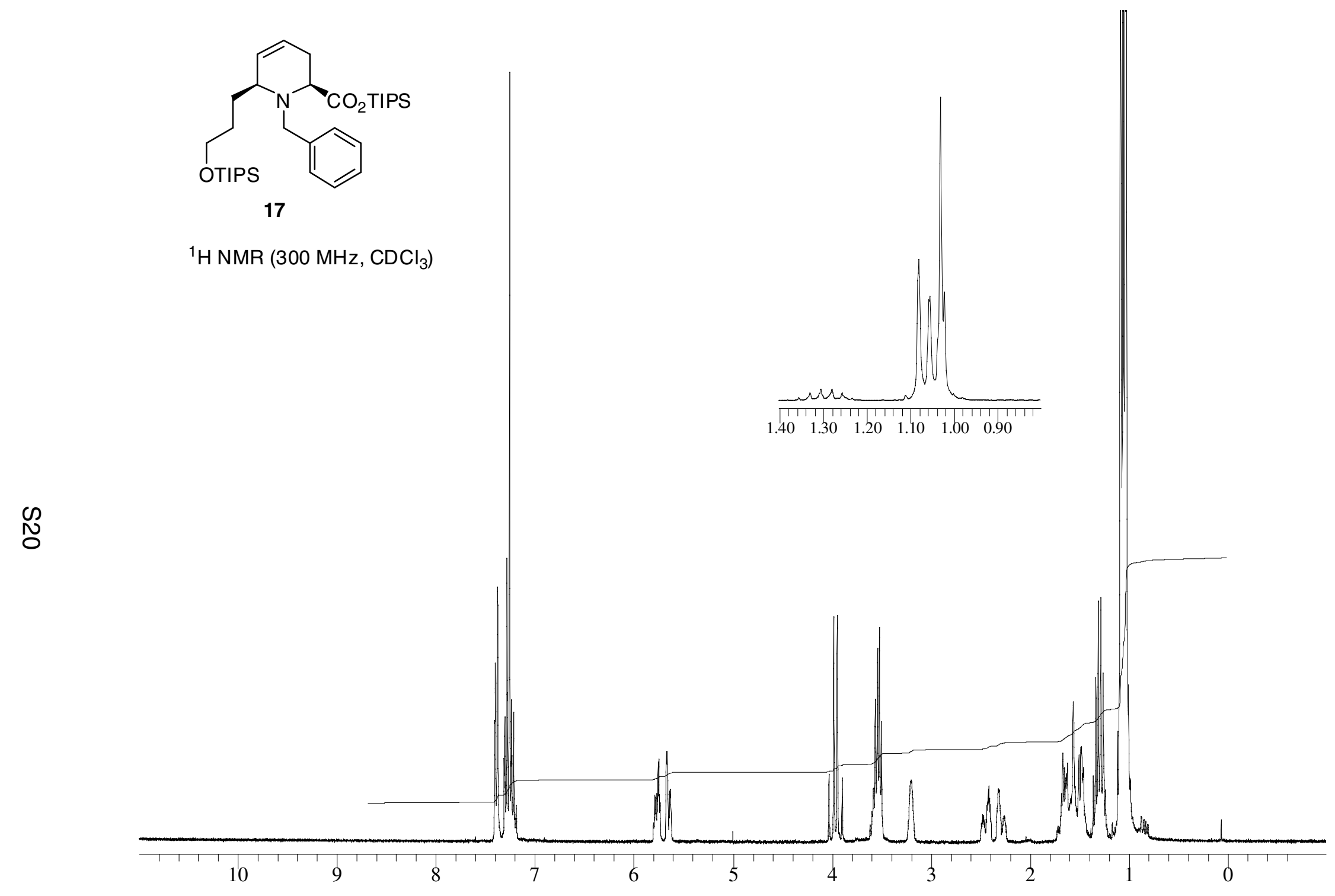




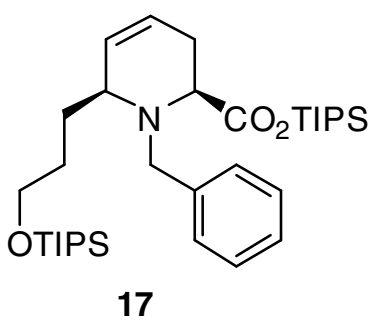

${ }^{13} \mathrm{C}$ NMR $\left(75 \mathrm{MHz}, \mathrm{CDCl}_{3}\right)$

N

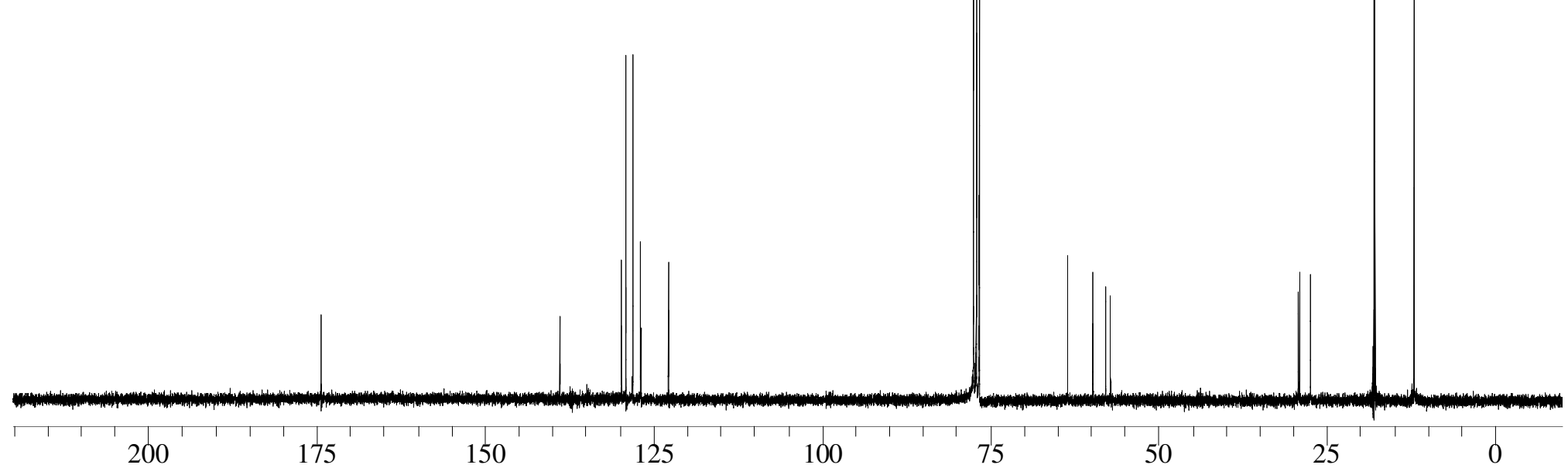




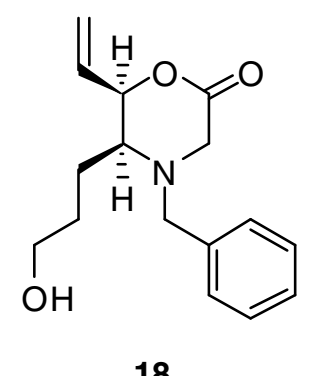

${ }^{1} \mathrm{H}$ NMR $\left(300 \mathrm{MHz}, \mathrm{CDCl}_{3}\right)$

N

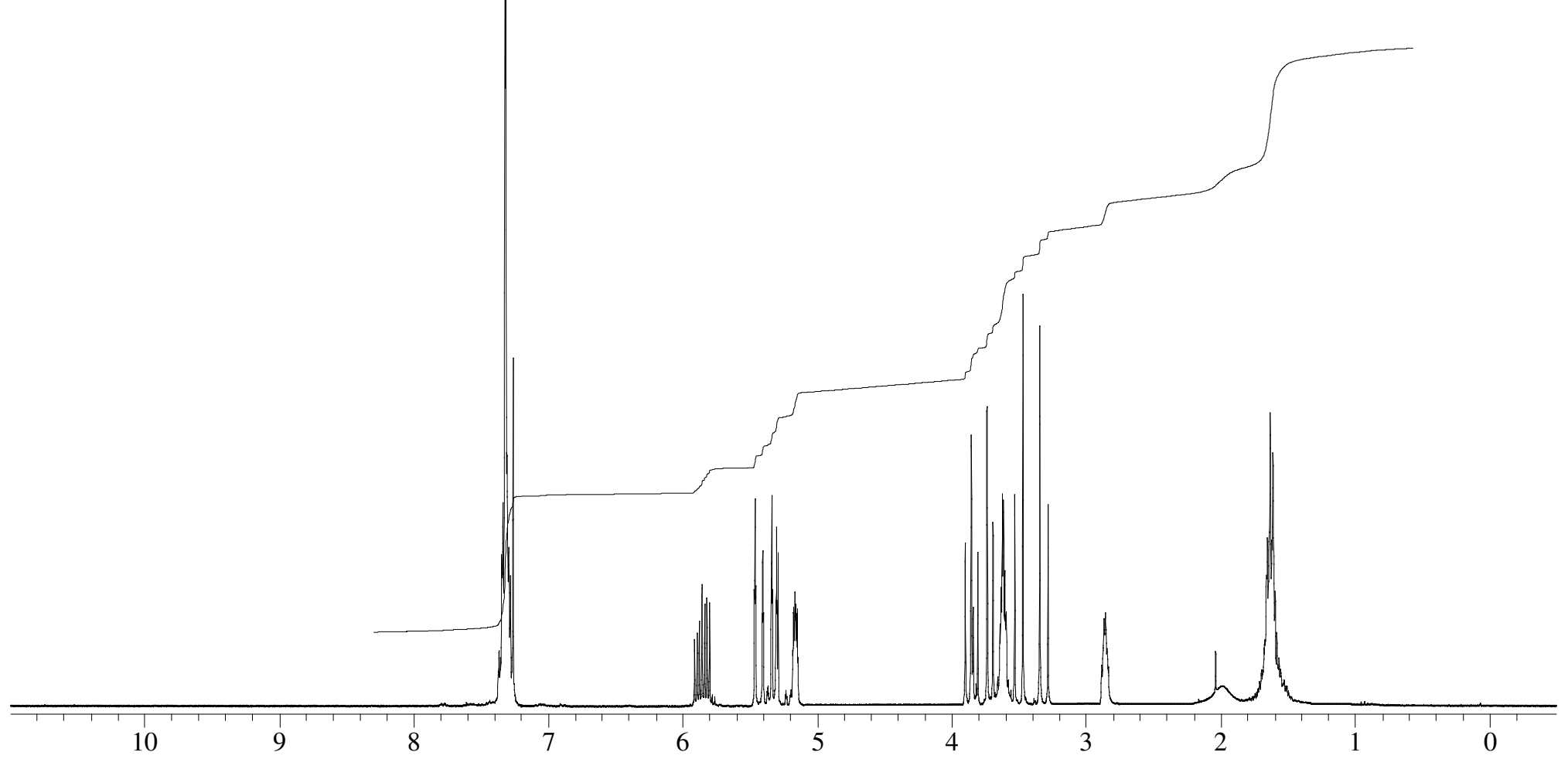






18

${ }^{1} \mathrm{H}$ NMR $\left(300 \mathrm{MHz}, \mathrm{CDCl}_{3}\right)$

స్ట
$\mathrm{H}^{\mathrm{a}}$

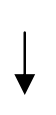

I=3.074
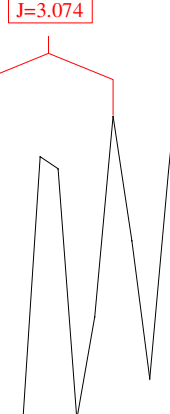

$\mathrm { J } = 5 . 7 0 9 \longdiv { \mathrm { J } = 1 . 3 1 8 }$




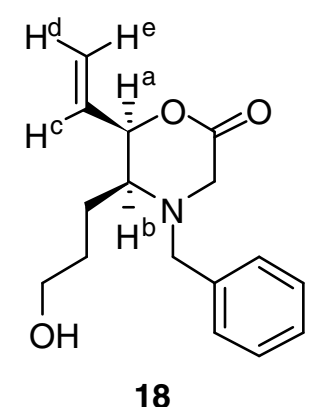

\section{Decoupling study of 18}

$\stackrel{\oplus}{N}$

${ }^{1} \mathrm{H}$ NMR $\left(300 \mathrm{MHz}, \mathrm{CDCl}_{3}\right)$

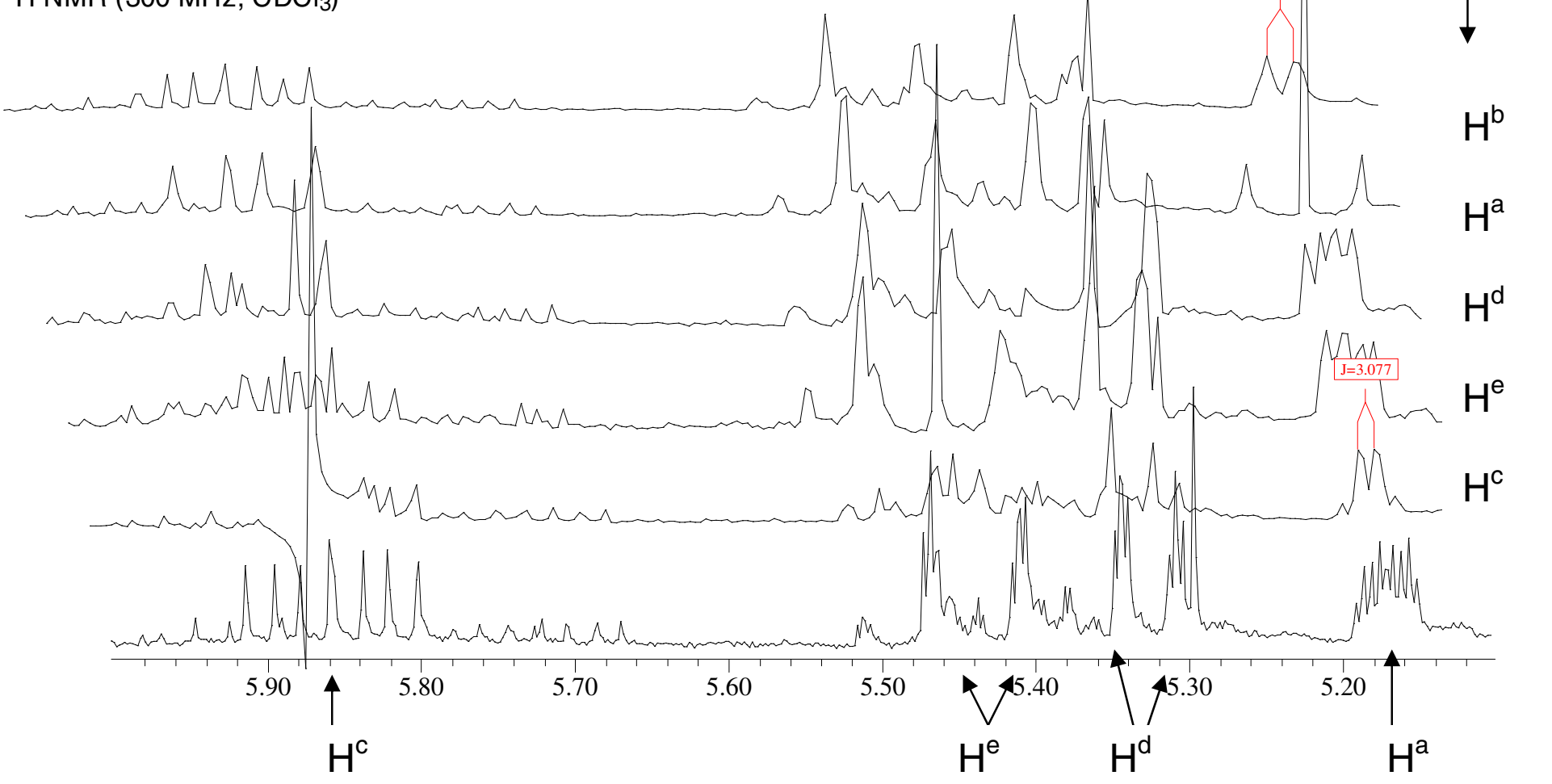




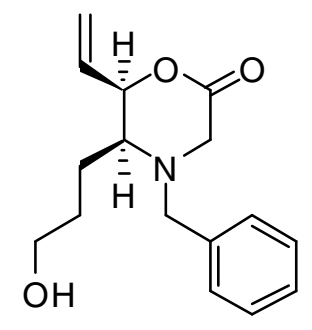

18

${ }^{13} \mathrm{C}$ NMR $\left(75 \mathrm{MHz}, \mathrm{CDCl}_{3}\right)$

N

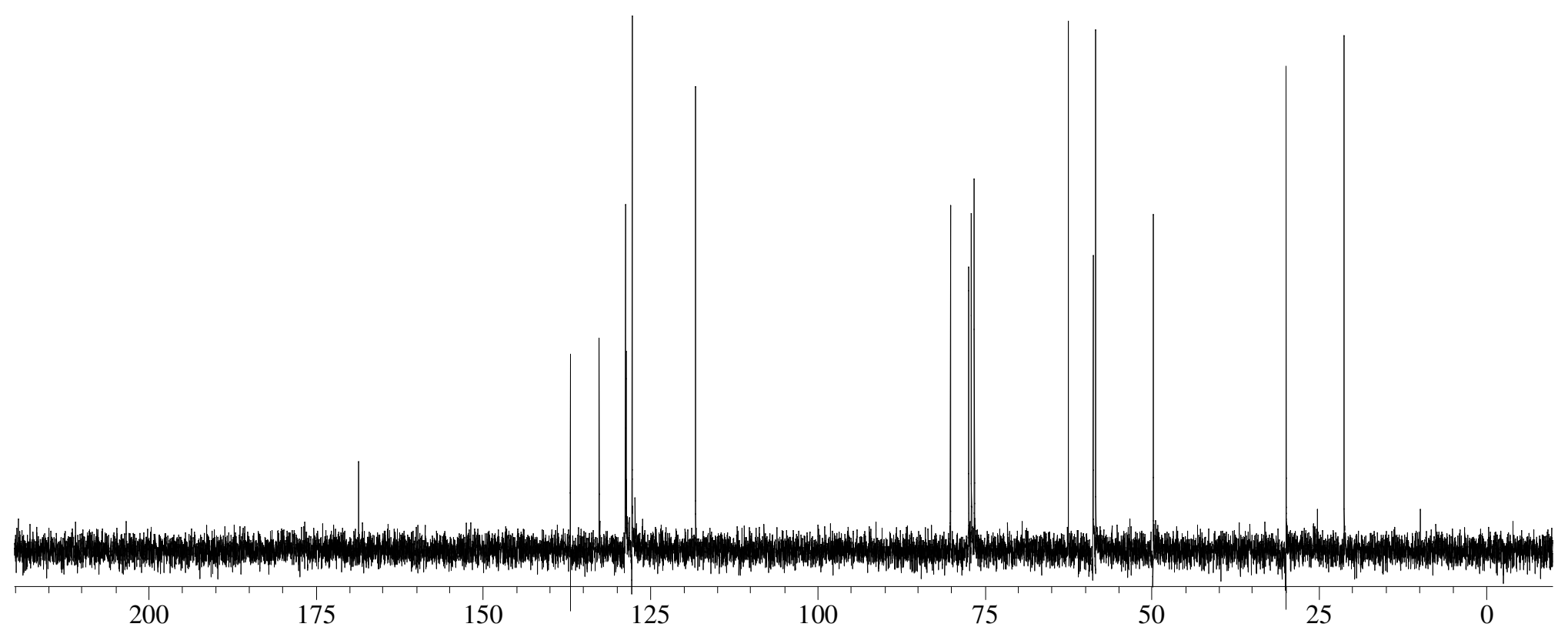




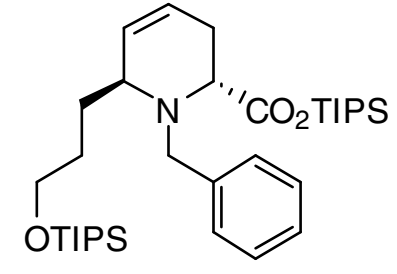

20

${ }^{1} \mathrm{H}$ NMR $\left(300 \mathrm{MHz}, \mathrm{CDCl}_{3}\right)$
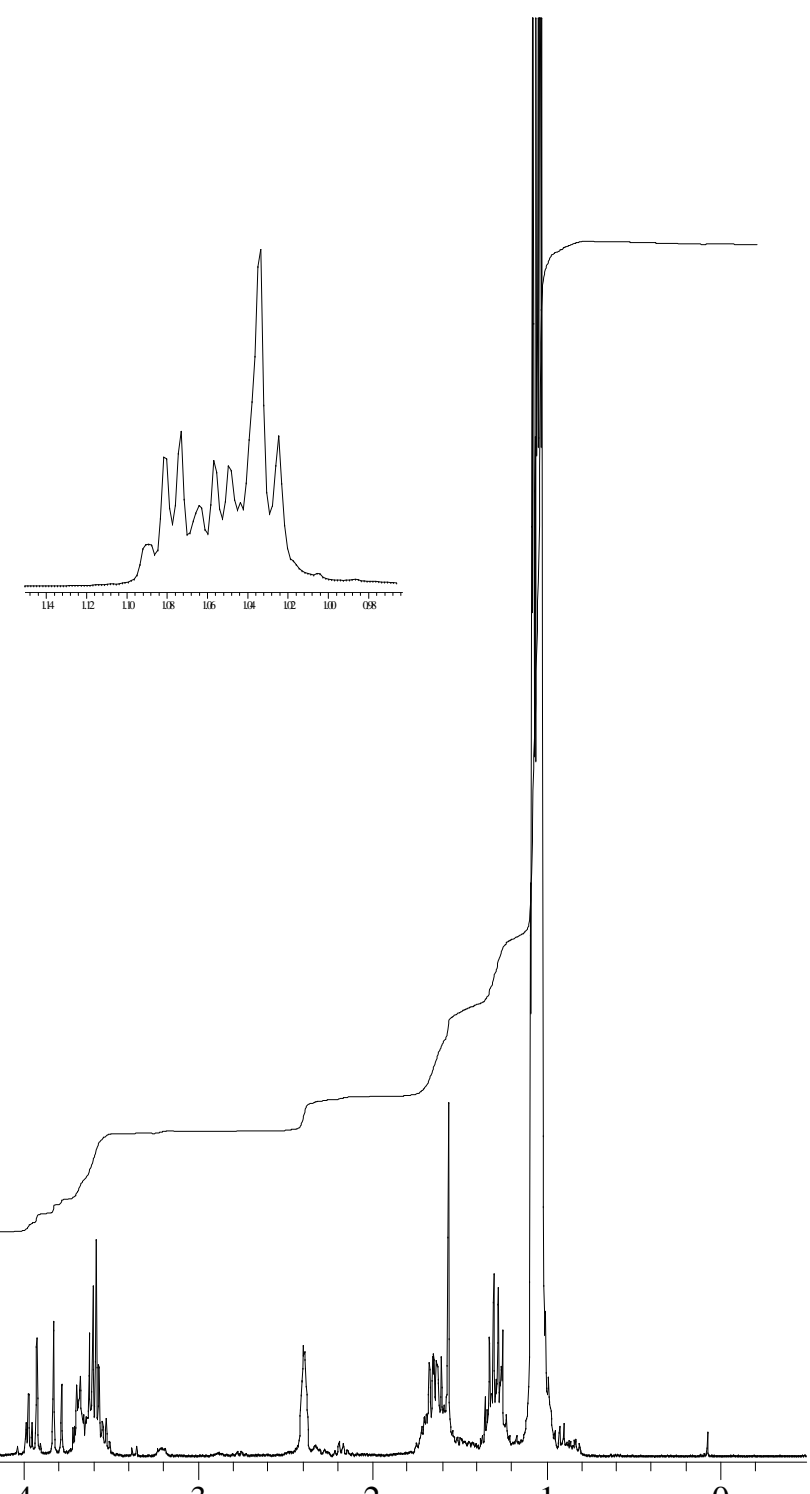

N్

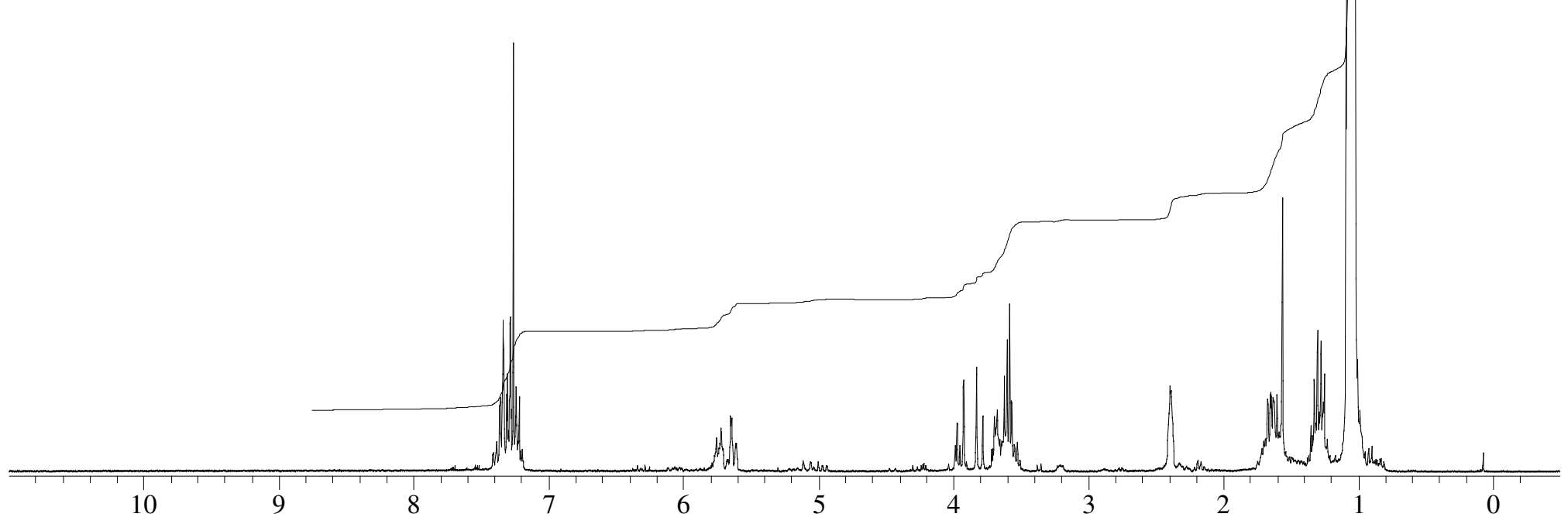




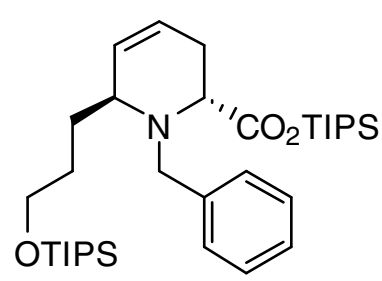

20

${ }^{13} \mathrm{C}$ NMR $\left(75 \mathrm{MHz}, \mathrm{CDCl}_{3}\right)$

N

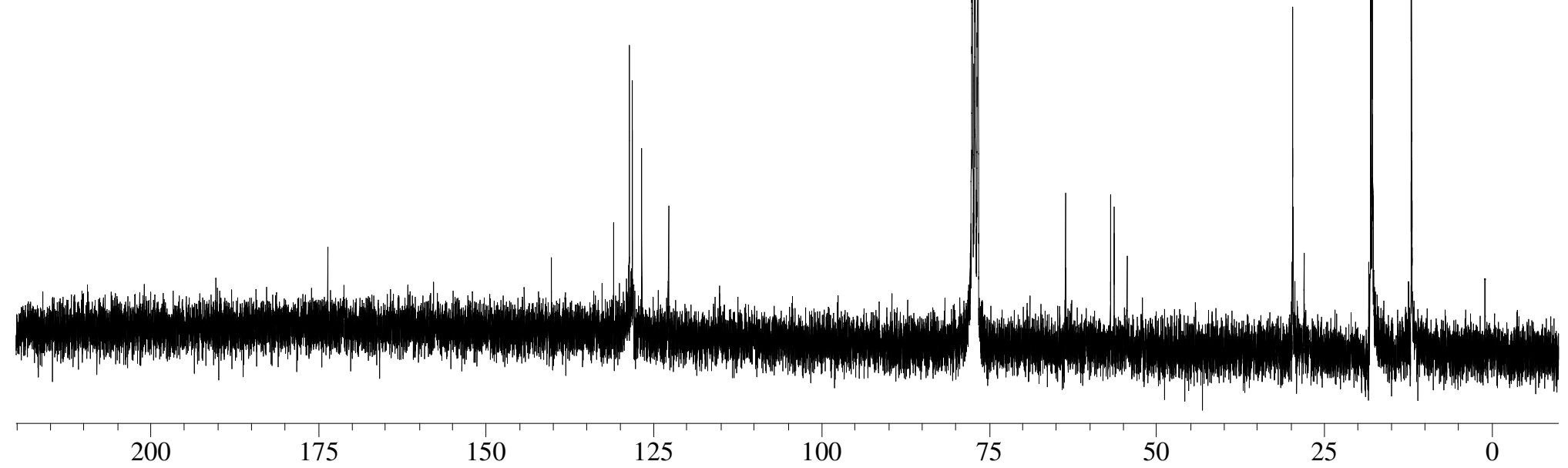




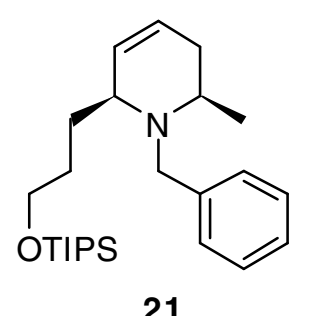

${ }^{1} \mathrm{H}$ NMR (300 MHz, $\left.\mathrm{CDCl}_{3}\right)$

N

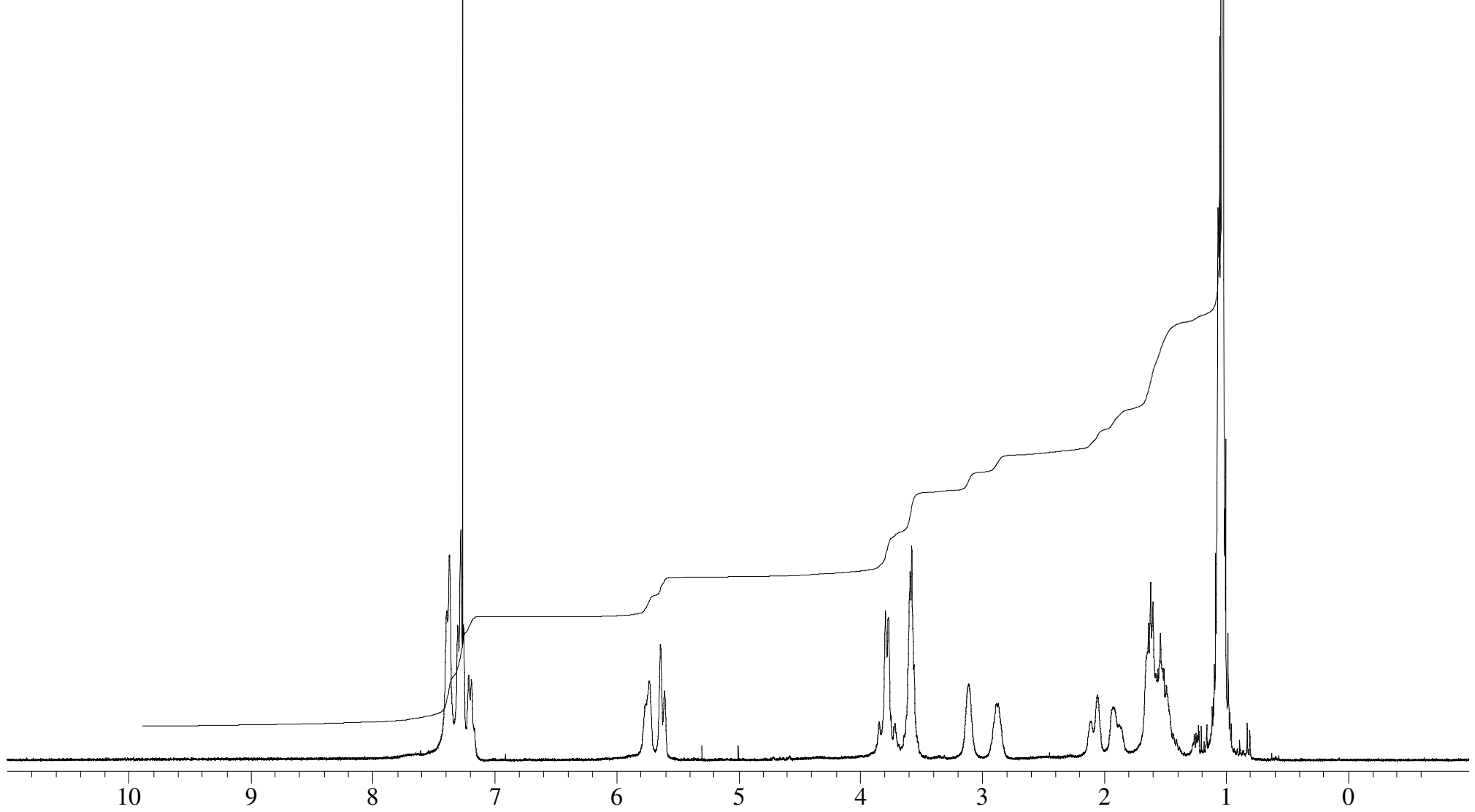




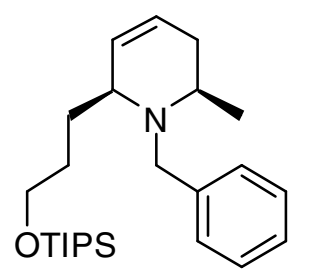

21

${ }^{13} \mathrm{C}$ NMR $\left(75 \mathrm{MHz}, \mathrm{CDCl}_{3}\right)$

N

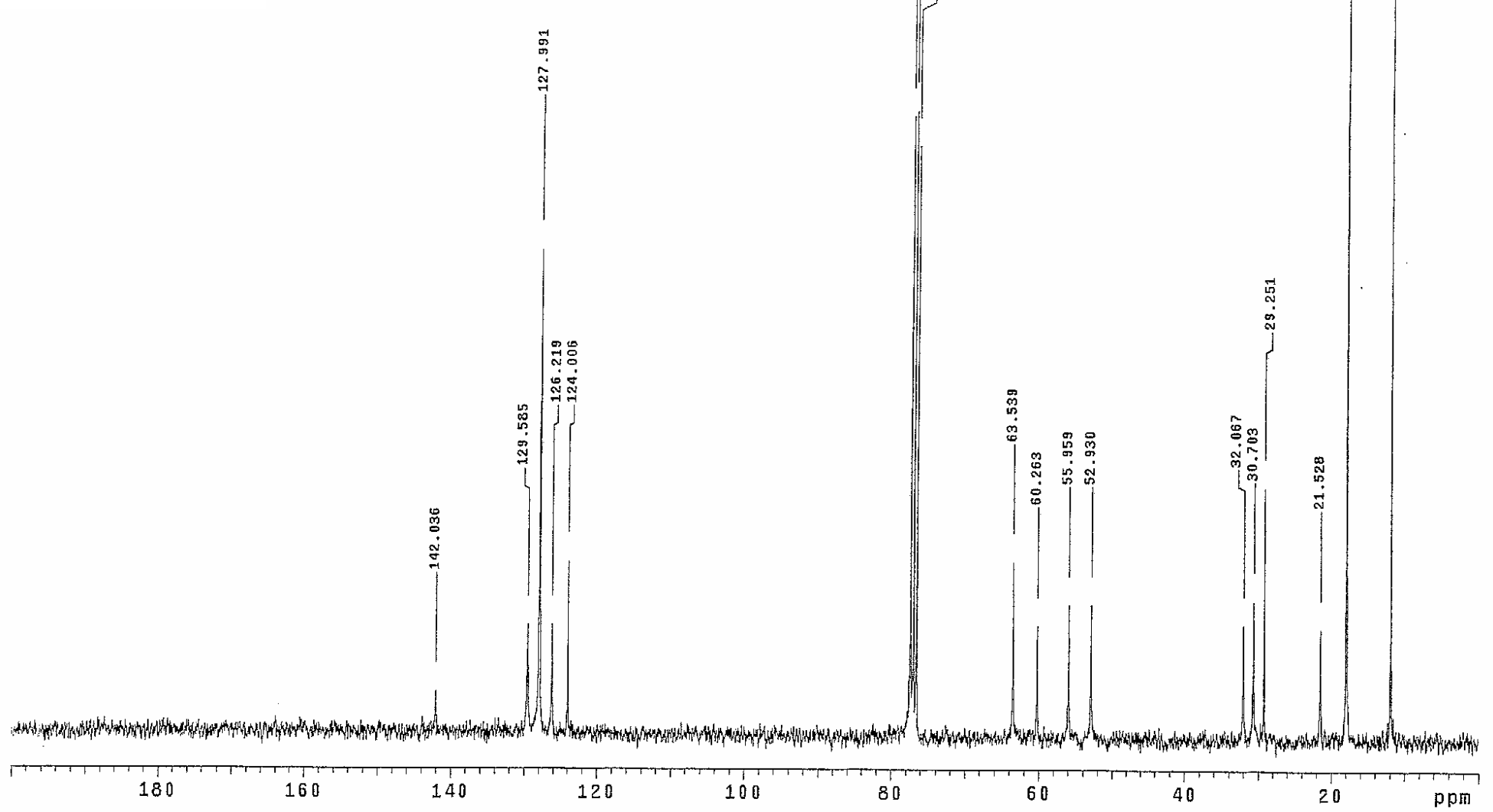




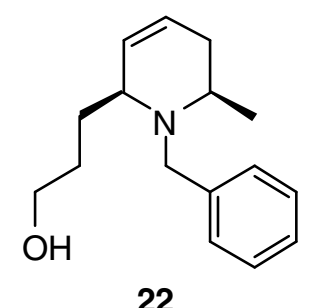

${ }^{1} \mathrm{H}$ NMR (300 MHz, $\mathrm{CDCl}_{3}$ )

$\mathscr{\mathscr { L }}$

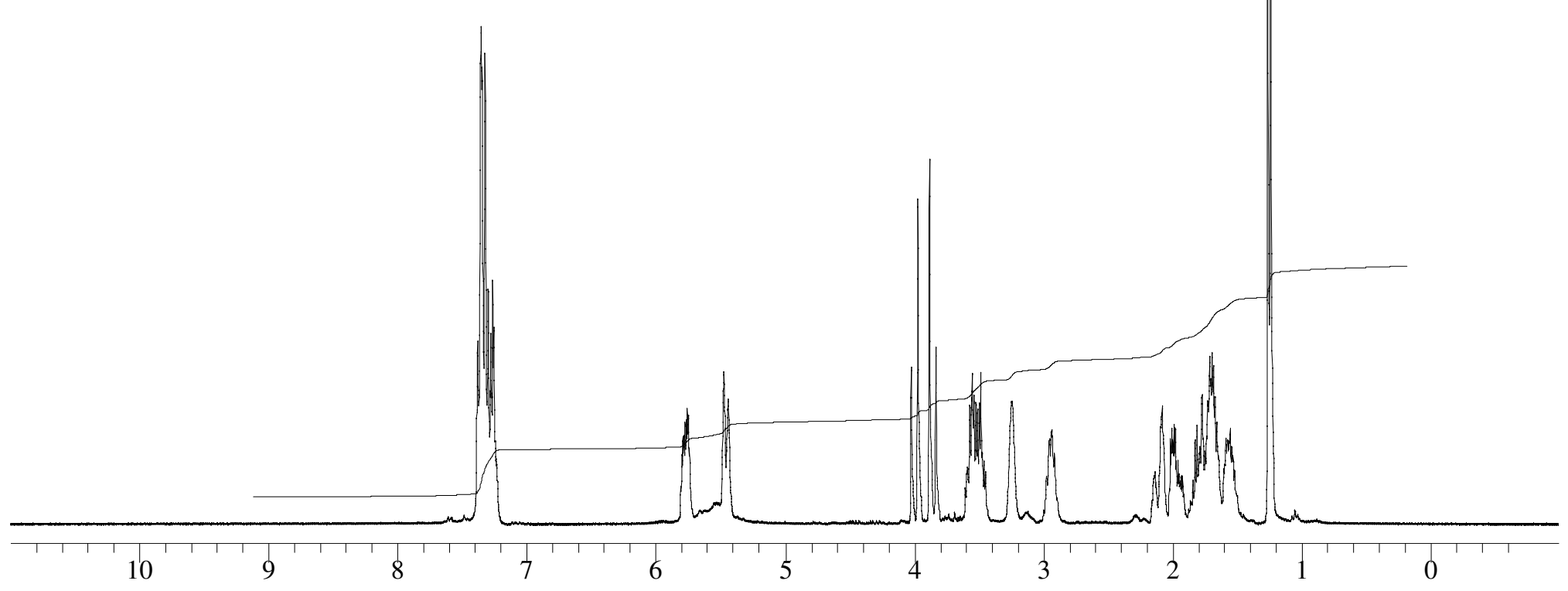




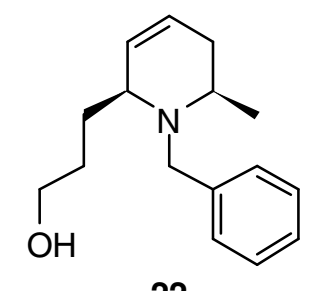

22

${ }^{13} \mathrm{C}$ NMR $\left(75 \mathrm{MHz}, \mathrm{CDCl}_{3}\right)$

$\stackrel{\mathscr{E}}{+}$

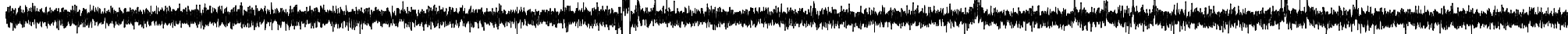

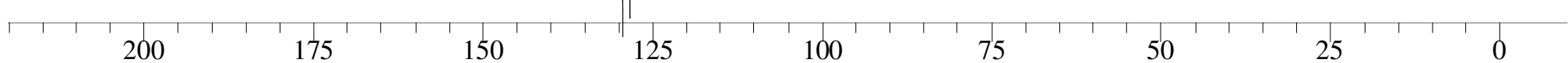




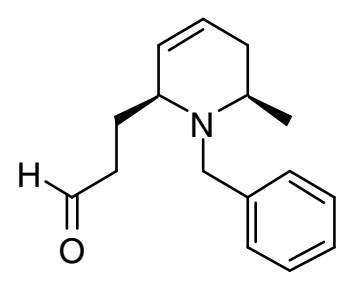

23

${ }^{1} \mathrm{H}$ NMR $\left(300 \mathrm{MHz}, \mathrm{CDCl}_{3}\right)$

డ్ల

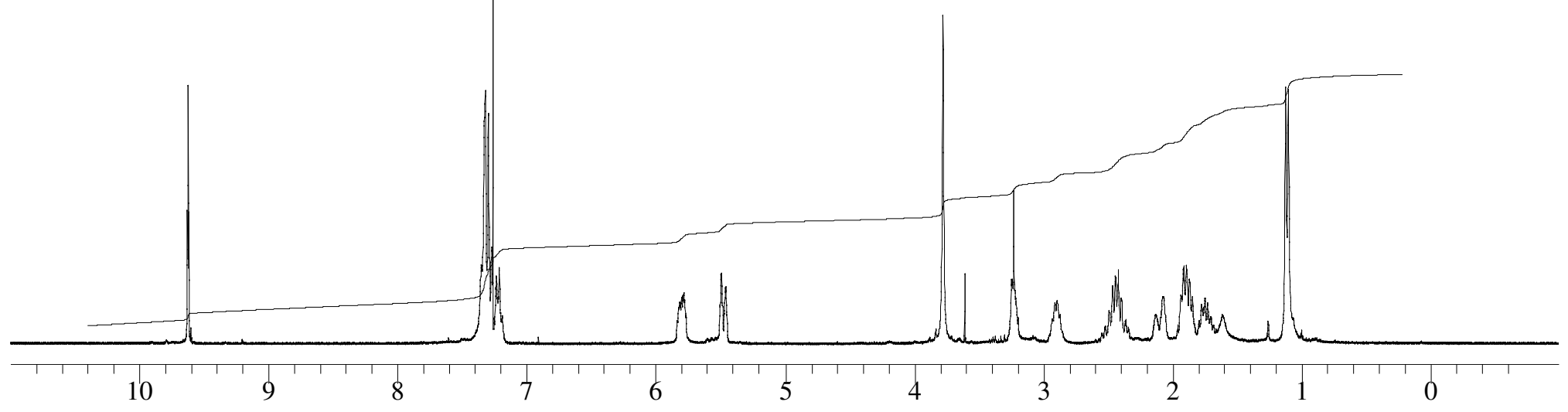




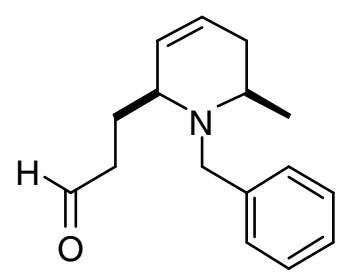

23

${ }^{13} \mathrm{C}$ NMR $\left(75 \mathrm{MHz}, \mathrm{CDCl}_{3}\right)$

$\stackrel{\mathscr{\omega}}{\mathscr{W}}$

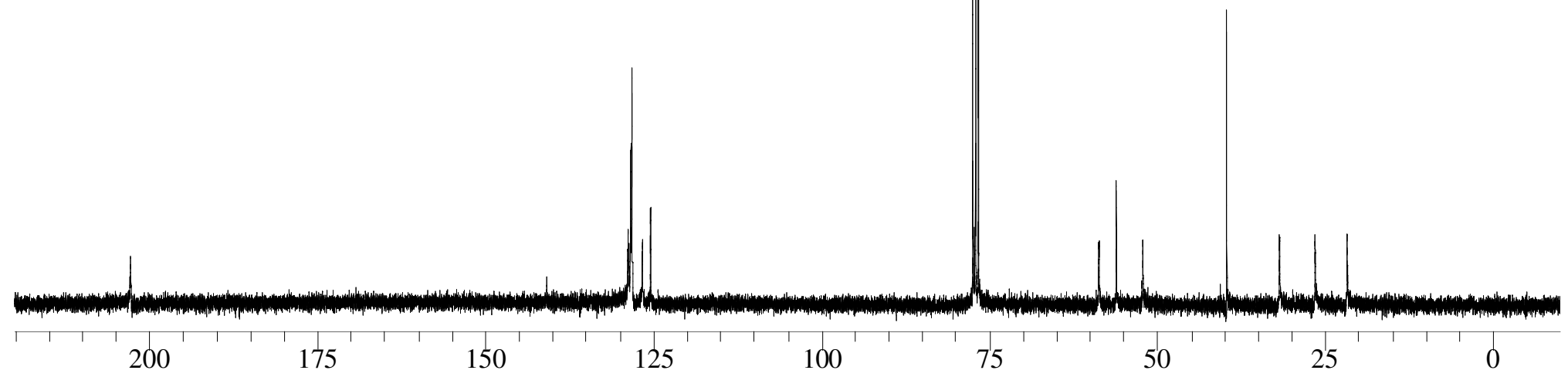


<smiles>COC(CC[C@@H]1C=CCC(C)N1Cc1ccccc1)OC</smiles>

24

${ }^{1} \mathrm{H}$ NMR $\left(300 \mathrm{MHz}, \mathrm{CDCl}_{3}\right)$

$\underset{\perp}{\mathscr{\perp}}$

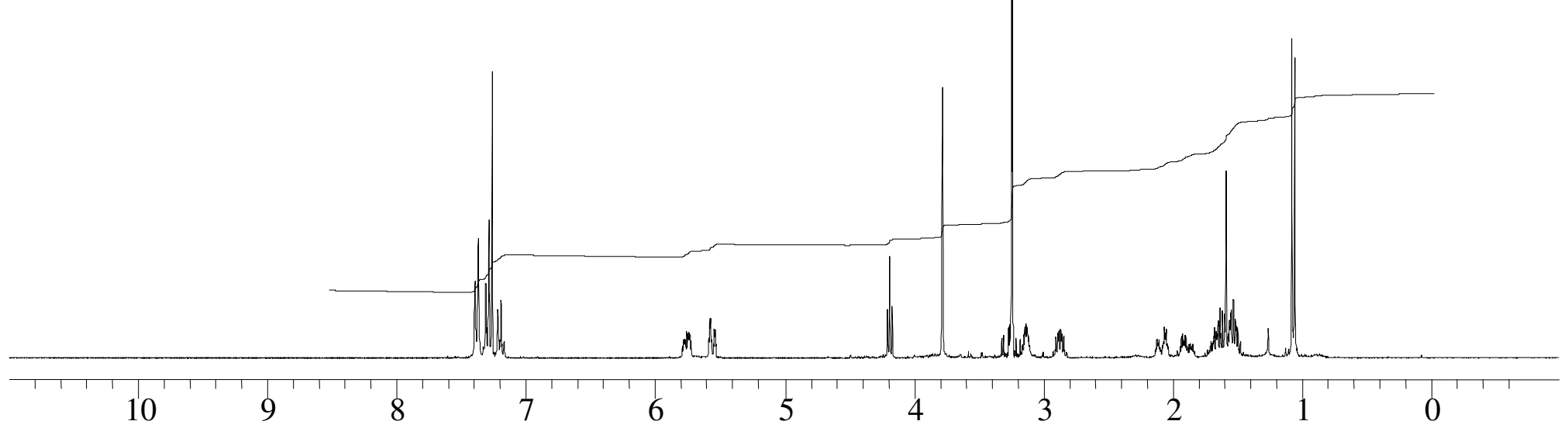




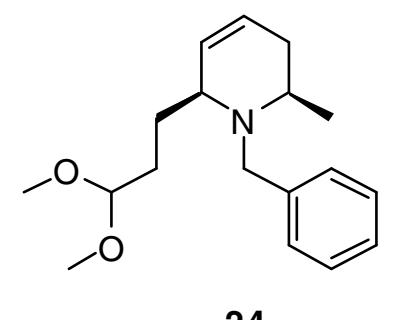

24

${ }^{13} \mathrm{C}$ NMR $\left(75 \mathrm{MHz}, \mathrm{CDCl}_{3}\right)$

W్

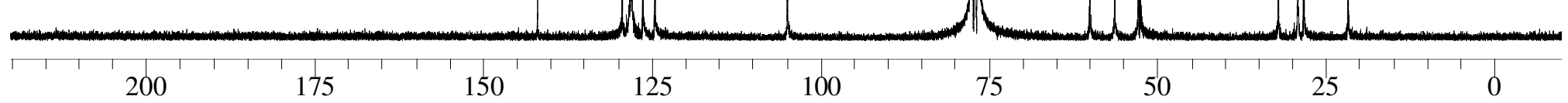




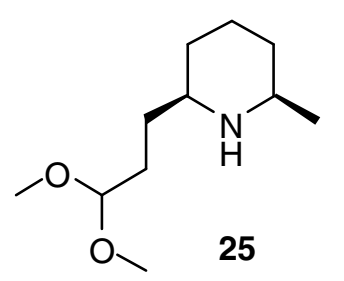

${ }^{1} \mathrm{H}$ NMR (300 MHz, $\left.\mathrm{CDCl}_{3}\right)$

$\mathscr{W}$

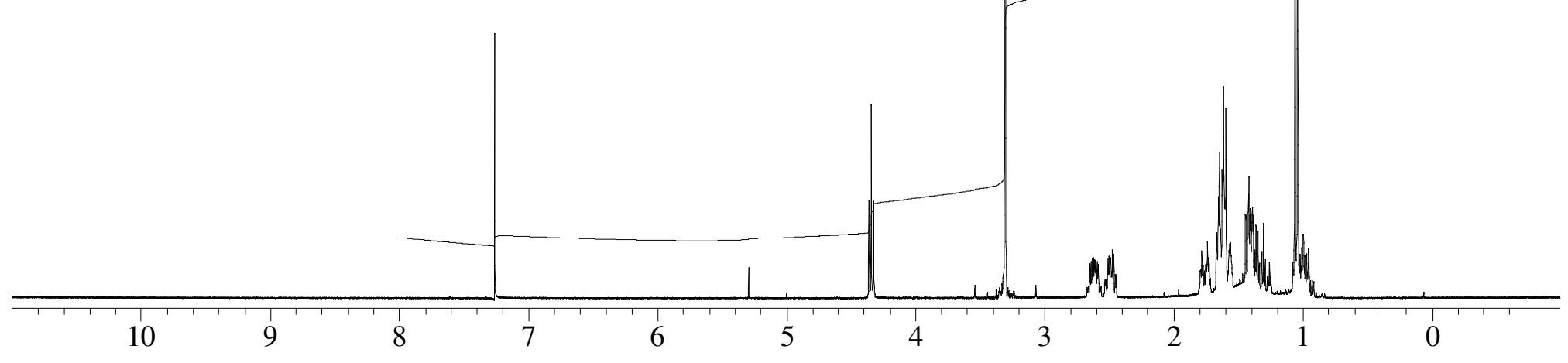




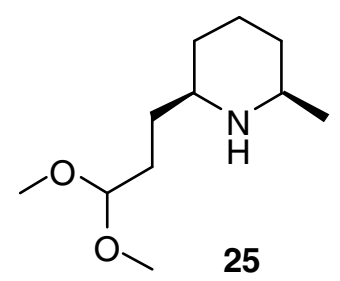

${ }^{13} \mathrm{C}$ NMR $\left(75 \mathrm{MHz}, \mathrm{CDCl}_{3}\right)$

W

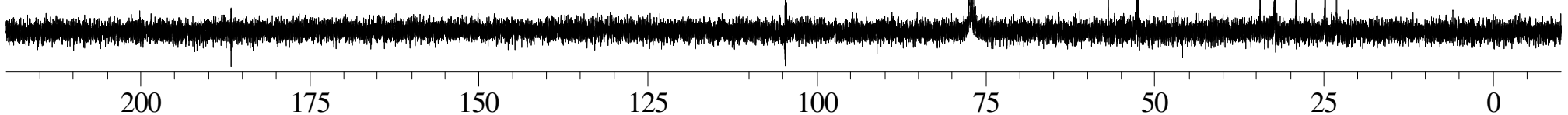




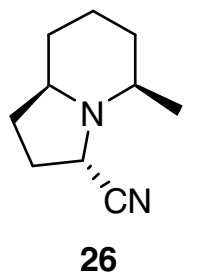

${ }^{1} \mathrm{H}$ NMR (300 MHz, CDCl 3 )

$\stackrel{\infty}{\infty}$

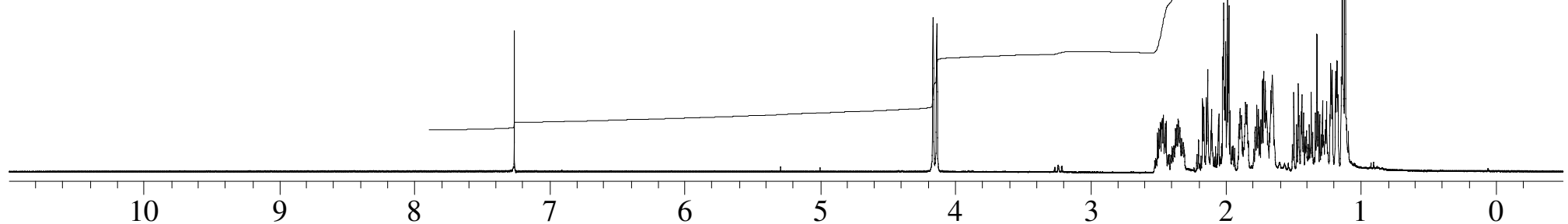




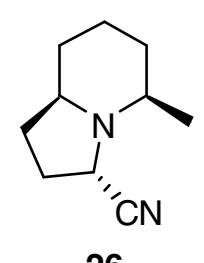

26

${ }^{13} \mathrm{C}$ NMR $\left(75 \mathrm{MHz}, \mathrm{CDCl}_{3}\right)$

W్

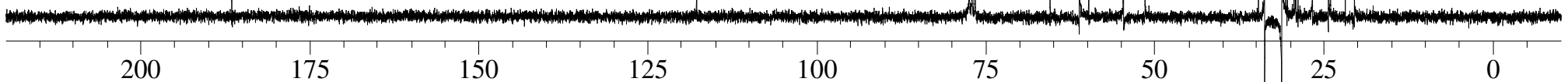




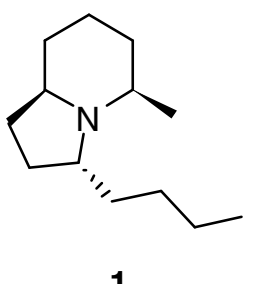

(-)-Indolizidine 195B

${ }^{1} \mathrm{H}$ NMR $\left(300 \mathrm{MHz}, \mathrm{CDCl}_{3}\right.$ )

D

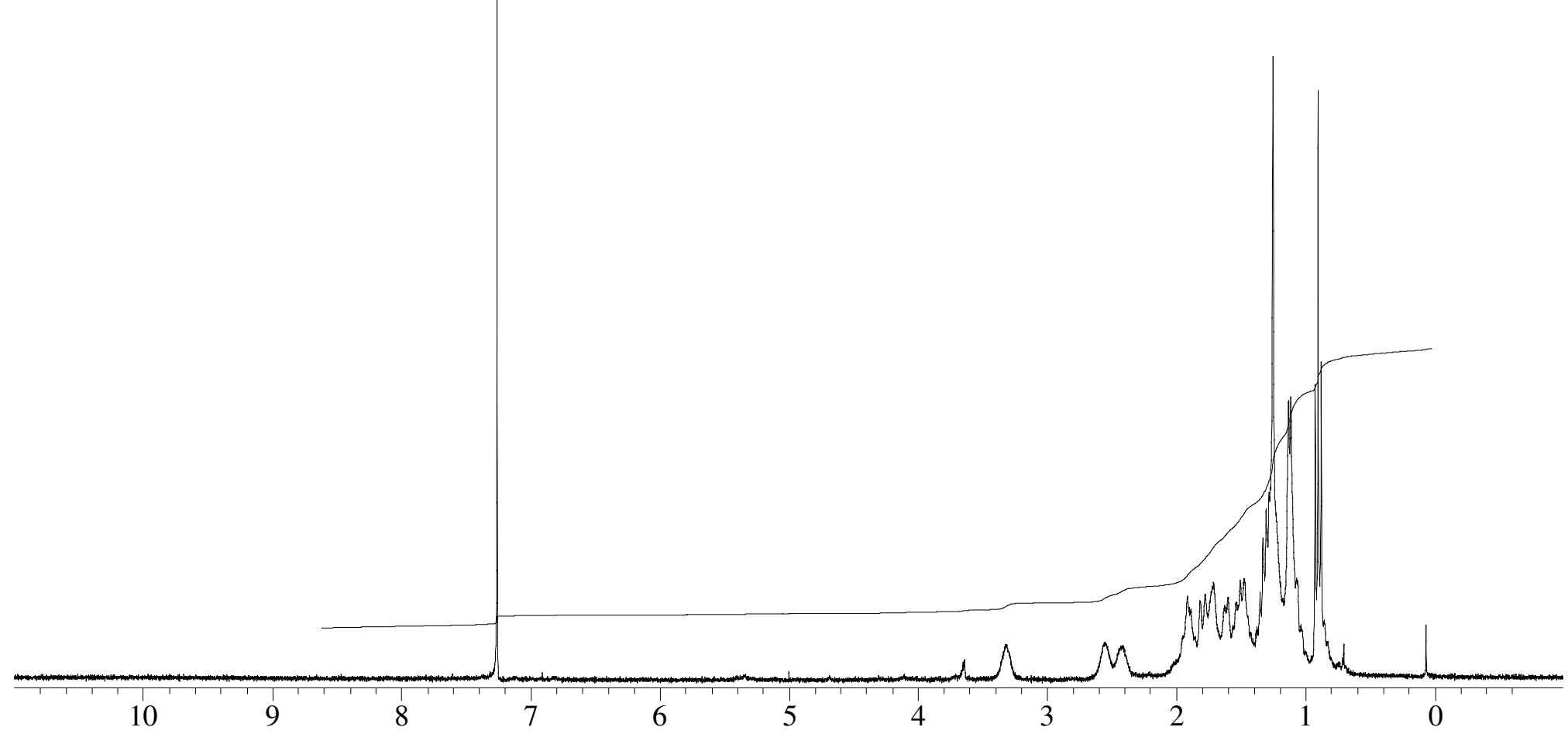


Diastereomer ratio of the mixture measured by integration of $\mathrm{H}^{\mathrm{a}}$ and $\mathrm{H}^{\mathrm{b}}$ signal of $1 \mathrm{H} \mathrm{NMR}$

$\stackrel{\infty}{ \pm}$

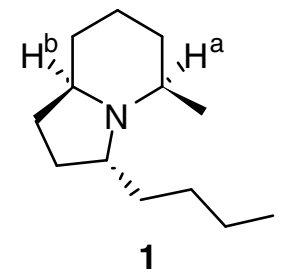

(-)-Indolizidine 195B

(-)-monomorine

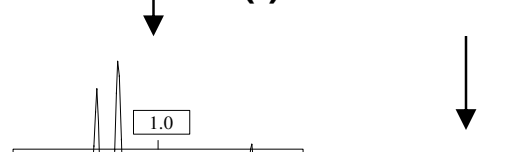

2.40

2.20

2.00

1.80 


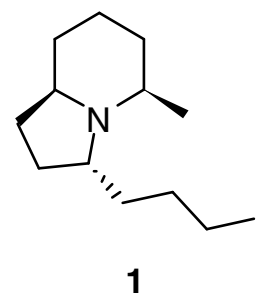

(-)-Indolizidine 195B

$\left.{ }^{13} \mathrm{C} \mathrm{NMR} \mathrm{(75} \mathrm{MHz,} \mathrm{CDCl}_{3}\right)$

N

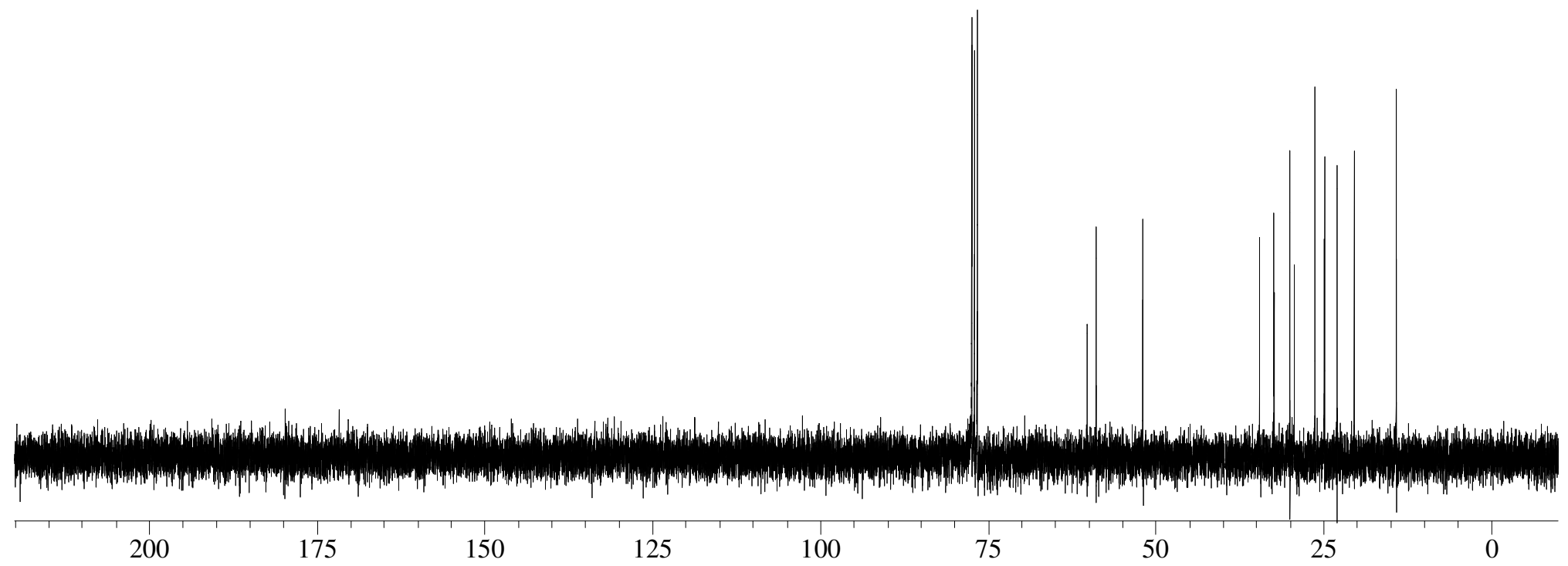

